\title{
Upper ionosphere of Mars is not axially symmetrical
}

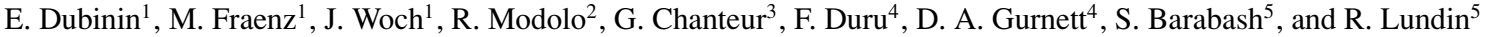 \\ ${ }^{1}$ MPI für Sonnensystemforschung, 37191 Katlenburg-Lindau, Germany \\ ${ }^{2}$ LATMOS, UVSQ-CNRS-IPSL, Guyancourt, France \\ ${ }^{3}$ Ecole Polytechnique-LPP, Palaiseau, France \\ ${ }^{4}$ Department of Physics and Astronomy, Iowa University, Iowa, USA \\ ${ }^{5}$ Swedish Institute of Space Physics, Kiruna, Sweden
}

(Received February 4, 2011; Revised May 6, 2011; Accepted May 18, 2011; Online published March 8, 2012)

\begin{abstract}
The measurements carried out by the ASPERA-3 and MARSIS experiments on board the Mars Express (MEX) spacecraft show that the upper Martian ionosphere $(h \geq 400 \mathrm{~km})$ is strongly azimuthally asymmetrical. There are several factors, e.g., the crustal magnetization on Mars and the orientation of the interplanetary magnetic field (IMF) which can give rise to formation of ionospheric swells and valleys. It is shown that expansion of the ionospheric plasma along the magnetic field lines of crustal origin can produce bulges in the plasma density. The absense of a magnetometer on MEX makes the retrieval of an asymmetry caused by the IMF more difficult. However hybrid simulations give a hint that the ionosphere in the hemisphere $\left(E^{-}\right)$to which the motional electric field is pointed occurs more inflated than the ionosphere in the opposite $\left(E^{+}\right)$hemisphere.
\end{abstract}

Key words: Mars, ionosphere, crustal magnetization, solar wind.

\section{Introduction}

The Martian ionosphere, formed by the photoionization of the major neutral constituents $\mathrm{CO}_{2}$ and $\mathrm{O}$ with subsequent molecular reactions creating $\mathrm{O}_{2}^{+}$as the major ionospheric ion species and $\mathrm{O}^{+}$becoming comparable at altitudes $\geq 300 \mathrm{~km}$, was extensively studied by radio occultation measurements (Kliore, 1992; Mendillo et al., 2004; Pätzold et al., 2005), radar remote sounding (Gurnett et al., 2005, 2008), in-situ measurements by retarding potential analyzer (Hanson et al., 1977; Hanson and Mantas, 1988) and 'plasma wave diagnostics' (Gurnett et al., 2005, 2008). Since Mars is not screened by a large-scale magnetic field the solar wind has direct access to the ionosphere providing momentum and energy transfer to the upper layers of the ionospheric plasma. While the ionosphere at the heights $\leq 200 \mathrm{~km}$ is in photochemical equilibrium and the height profile and solar zenith dependence rather closely follow the Chapman model (Gurnett et al., 2008; Morgan et al., 2008; Withers, 2009; Lillis et al., 2010), deviations from the model become essential at larger altitudes. At altitude $300-550 \mathrm{~km}$ the median electron density ceases to follow $\sqrt{\cos (\mathrm{SZA})}$ dependence and remains almost constant for all solar zenith angles up to SZA $\sim 80^{\circ}$ (Duru et al., 2008).

It is reasonable to assume that dynamics of the upper ionosphere is strongly influenced by the interaction between solar wind and ionospheric plasma mediated by the IMF draped around the planet. This interaction can introduce asymmetry in the plasma distribution. Here we present data which show that in contrast to the low-altidude ionosphere,

Copyright (C) The Society of Geomagnetism and Earth, Planetary and Space Sciences (SGEPSS); The Seismological Society of Japan; The Volcanological Society of Japan; The Geodetic Society of Japan; The Japanese Society for Planetary Sciences; TERRAPUB.

doi:10.5047/eps.2011.05.022 which is almost axially symmetrical (e.g. the dawn-dusk asymmetry does not exceed 5-25\%, Lillis et al., 2010), the top-side ionosphere is very asymmetrical.

\section{Observations}

The MEX spacecraft is in a highly eccentric polar orbit around Mars with periapsis and apoapsis of about 275 and $10000 \mathrm{~km}$, respectively. The measurements were made by the ASPERA-3 (Analyzer of Space Plasma and Energetic Atoms) and MARSIS (Mars Advanced Radar for Subsurface and Ionospheric Sounding) experiments. ASPERA3 comprises two plasma sensors: the Ion Mass Analyzer (IMA) and ELectron Spectrometer (ELS) (Barabash et al., 2006). The Ion Mass Analyzer (IMA) determines the composition, energy, and angular distribution of ions in the energy range $\approx 10 \mathrm{eV}-30 \mathrm{keV}$. Mass $(m / q)$ resolution is provided by a combination of an electrostatic analyzer with deflection of ions in a cylindrical magnetic field set up by permanent magnets. In the energy range $\geq 50 \mathrm{eV}$, IMA measures fluxes of different $(\mathrm{m} / \mathrm{q})$ ion species with a time resolution of $\sim 3 \mathrm{~min}$ and a field of view of $90^{\circ} \times 360^{\circ}$ (electrostatic steering provides an elevation coverage of $\left.\pm 45^{\circ}\right)$. The measurements of the cold/low-energy component $(\leq 50 \mathrm{eV})$ are carried out without the elevation coverage, and therefore, the time-resolution of these measurements increases up to $12 \mathrm{~s}$. The ELS sensor measures $2 \mathrm{D}$ distributions of the electron fluxes in the energy range $1 \mathrm{eV}-20 \mathrm{keV}$ $(\delta E / E=8 \%)$ with a field of view of $4^{\circ} \times 360^{\circ}$ and a time resolution of $\sim 4 \mathrm{~s}$.

The MARSIS radar sounder ( $f \approx 0.1$ to $5.5 \mathrm{MHz}$ ), designed to monitor the ionospheric height profile and the subsurface of the planet, consists of a $40 \mathrm{~m}$ tip-to-tip electric dipole antenna, a radio transmitter, a receiver, and a digital signal processing system. For the normal iono- 

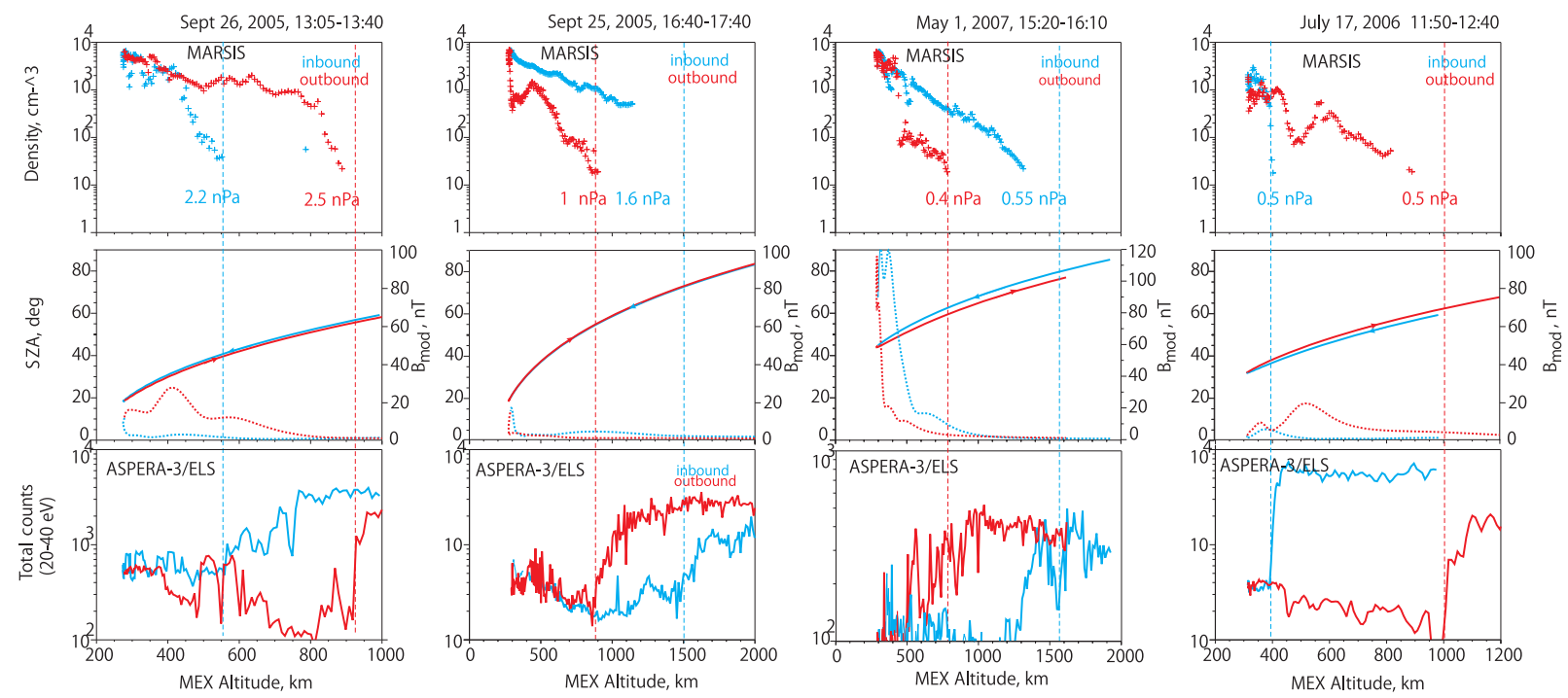

Fig. 1. From top to bottom: (a) height profiles of the electron number density measured by MARSIS, blue and red symbols correspond to the measurements made on the inbound and outbound orbital legs, respectively; (b) solar zenith angles (solid curves) and the value of the model crustal magnetic field; (c) fluxes of 20-40 eV electrons. Dashed vertical lines show the positions of the induced magnetospheric boundary on inbound and oubound orbital pathes determined from the drop of fluxes of the magnetosheath-like electrons.

spheric sounding mode used by MARSIS the transmitter steps through 160 frequencies $(\Delta f / f \approx 2 \%)$ from $100 \mathrm{kHz}$ to $5.5 \mathrm{MHz}$. The receiver has a bandwidth of $10.9 \mathrm{kHz}$ centered on the frequency of the transmitted pulse. A complete scan through all 160 frequencies takes $1.26 \mathrm{~s}$, and the basic sweep cycle is repeated once every $7.54 \mathrm{~s}$. In addition to remote radio sounding, the local electron density and the magnetic field strength can also be retrieved from the MARSIS data by measuring the frequencies of local electron plasma oscillations and their harmonics and electron cyclotron waves excited by the radar transmitter in the nearby plasma. These measurements were made in the region around periapsis, at the altitudes $\leq 1300 \mathrm{~km}$ (Gurnett et al., 2005, 2008; Duru et al., 2008).

Figure 1 shows examples of the height profiles of the electron number density inferred from the in-situ MARSIS measurements of the local electron plasma frequency along the MEX orbits (top panels). Blue and red curves show the data on the inbound and outbound legs, respectively. Solar wind dynamic pressure, evaluated from the ASPERA-3 measurements upstream of the inbound and outbound bow shock, as $n_{\mathrm{e}} m_{\mathrm{p}} V_{\mathrm{He}^{++}}^{2}$, where $n_{\mathrm{e}}, V_{\mathrm{He}^{++}}$and $m_{\mathrm{p}}$ are the electron number density, the bulk speed of alpha-particles in the solar wind and the proton mass, respectively, is also given. Solid curves on the middle panels present the corresponding solar zenith angles. Dotted curves depict the value of the model crustal magnetic field (Cain et al., 2003). It is observed that the altitude distribution of the ionospheric plasma is very asymmetrical although the spacecraft sampled the same solar zenith angles. Asymmetry becomes evident at $h \sim 400 \mathrm{~km}$ and reaches several hundreds $\mathrm{km}$. The bottom panels show the curves for integral fluxes of the electrons measured in the energy range $20-40 \mathrm{eV}$ by ELS/ASPERA-3. These fluxes of the magnetosheath electrons can be used to mark the position of the magnetospheric boundary (Dubinin et al., 2008a). It is seen that asymmetry in the height profiles of the ionospheric plasma
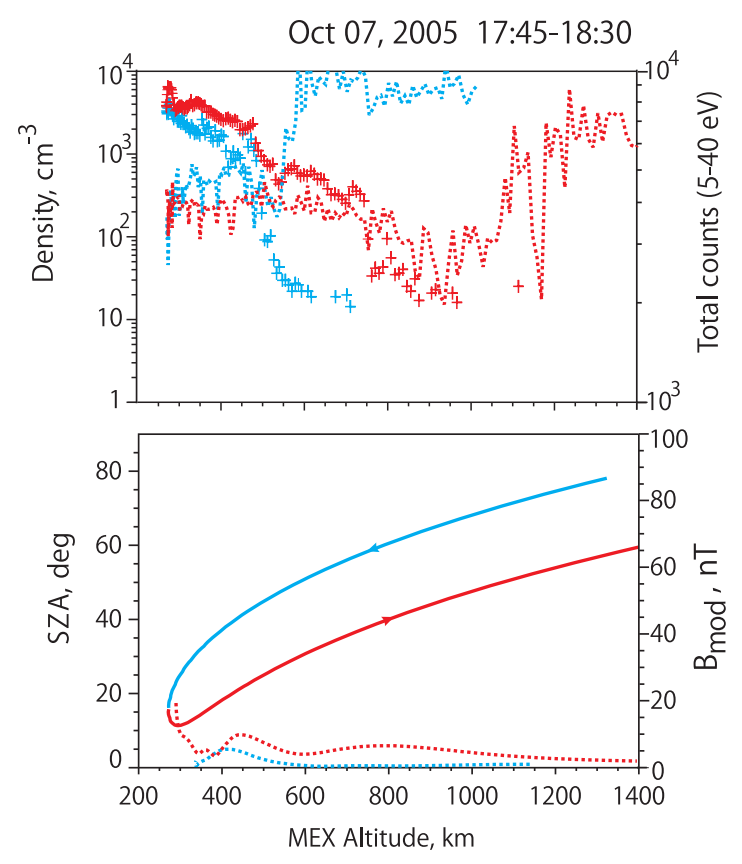

Fig. 2. Altitude profiles of the electron number density measured by MARSIS (symbols) and solar wind electron fluxes measured by ELS (dotted curves). Blue and red symbols correspond to the measurements made on the inbound and outbound orbital legs, respectively. The bottom panel depicts the solar zenith angle and the value of the crustal field.

density is closely related to the asymmetry of the solar wind flow around the planet-solar wind penetrates to different altitudes on inbound and outbound paths. Note also that although the crustal magnetic field at altitudes, at which asymmetry occurs, is rather weak, the ionosphere swells (shrinks) on the orbital paths where the field is stronger (weaker).

Figure 2 shows another example of the measurements made by MARSIS and ASPERA-3. Blue and red sym- 

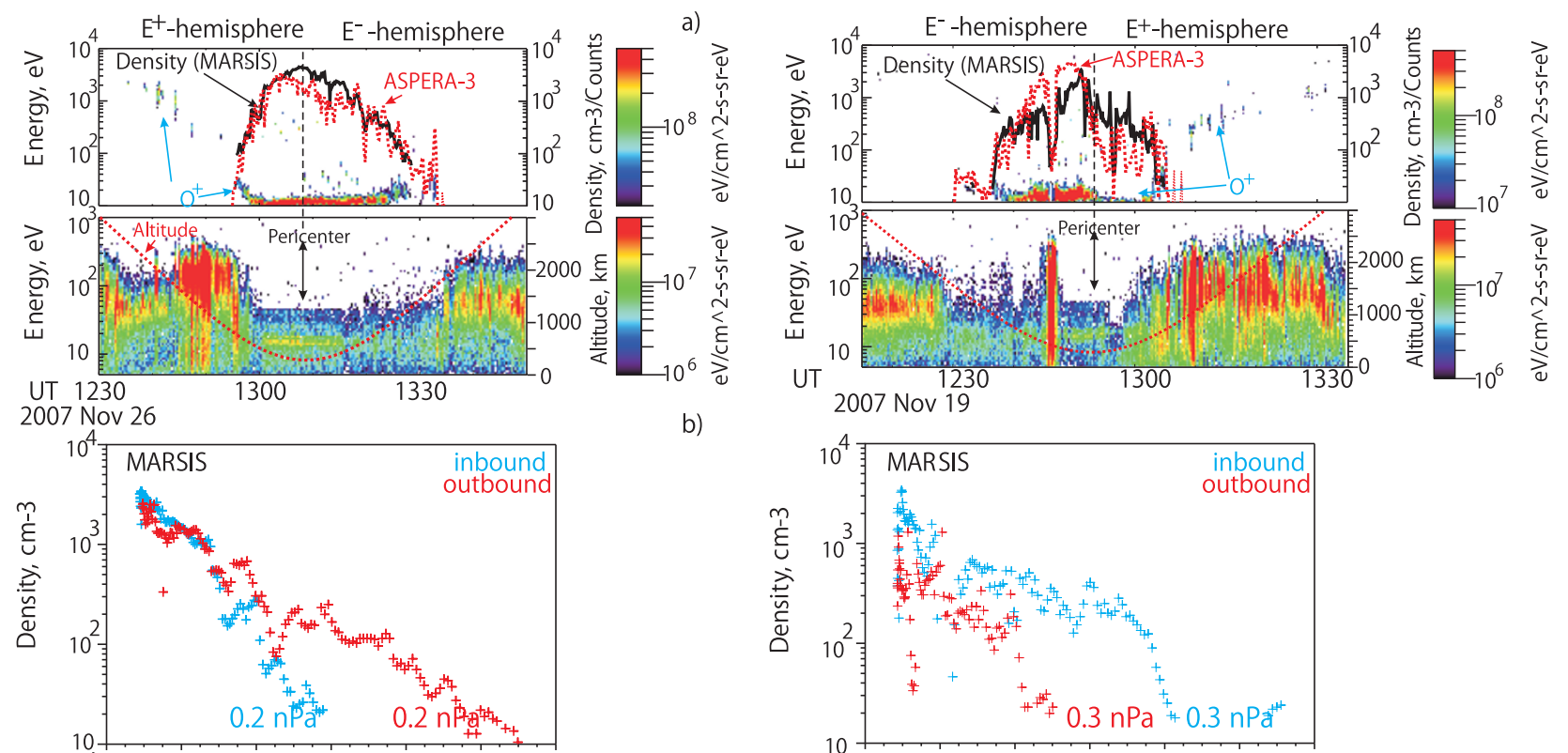

b)
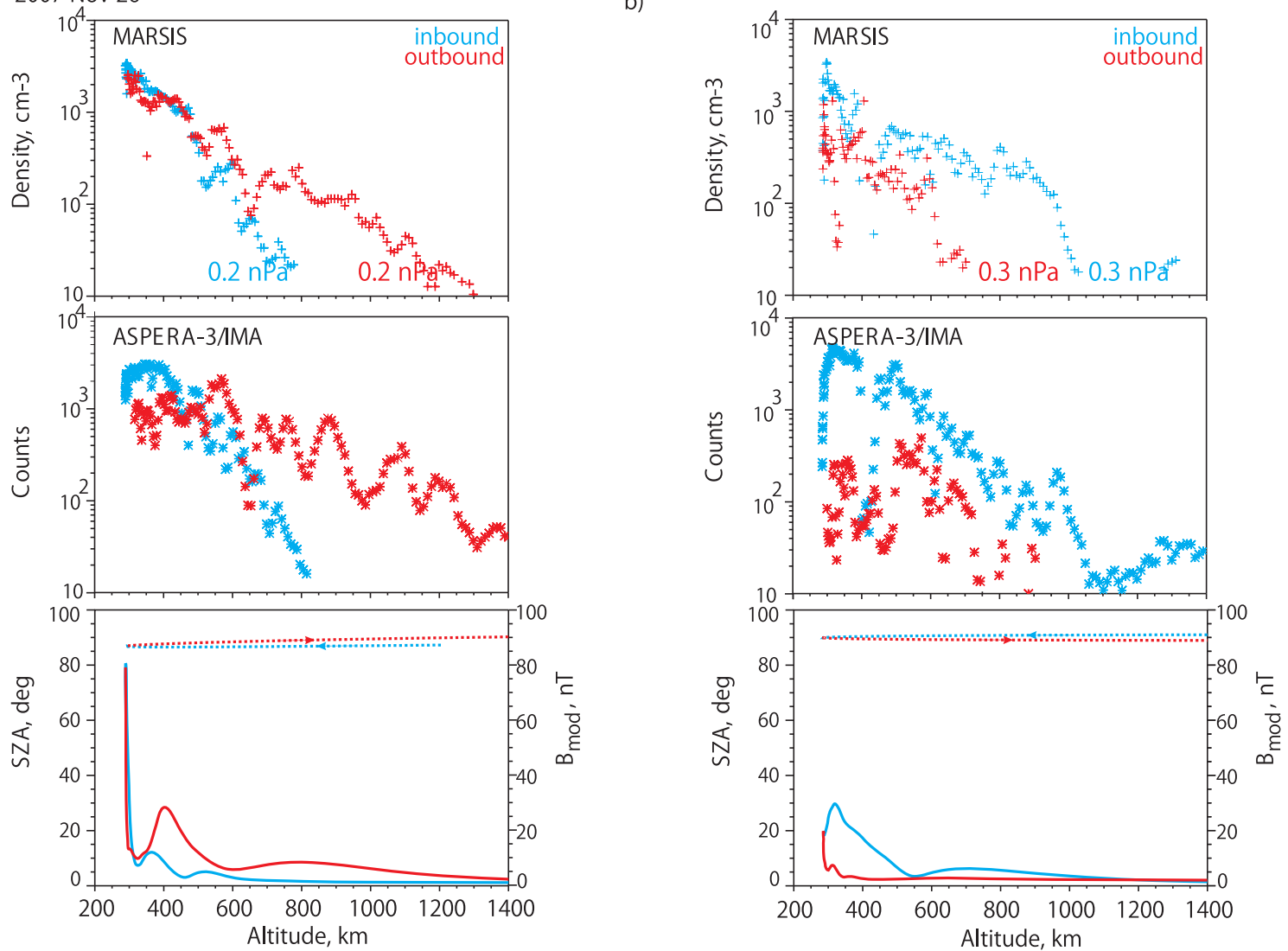

Fig. 3. From top to bottom: (a) energy-time spectrograms of oxygen ions; blue and red curves show the electron number density (MARSIS) and total number of counts in the 'oxygen channel' $\left(E_{\mathrm{i}} \leq 50 \mathrm{eV}\right)$. (b) energy-time spectrograms of electron fluxes; the dotted red curves show the altitude of MEX. (c) height profiles of the electron number densities on the inbound (blue) and outbound (red) orbital legs. Solar wind dynamic pressure corresponding to the inbound and outbound legs is also given. (d) height profiles of oxygen ions measured by IMA/ASPERA on the inbound and outbound parts of the MEX orbits. (e) solar zenith angle and the crustal magnetic field taken from the Cain model.

bols on the top panel present the electron number density (MARSIS) on the inbound and outbound orbital legs, respectively while the dotted curves show the corresponding height profiles of the electron fluxes (ELS). Although one would expect that the height of the ionosphere increases with the solar zenith angle, the ionosphere in this case expands to higher altitudes on the outbound path when MEX surveys smaller solar zenith angles than on the inbound part of the spacecraft trajectory.

Asymmetry of the upper ionosphere is also clearly observed from the IMA measurements of cold ionospheric ions which became available after May 1, 2007 when the new configuration of IMA was uploaded. Figure 3 depicts the MEX data along the orbits surveying the ionosphere in the terminator plane. Top panels present the energy-time spectrograms of oxygen ions. The low-energy
$\left(E_{\mathrm{i}} \sim 10 \mathrm{eV}\right)$ component corresponds to the bulk ionospheric plasma. In the interface region adjacent to the magnetosheath, the ionospheric plasma is transported tailward and its bulk speed increases with distance. It is worth noting that the measured energies of the ions are higher than the real values due to the negative value of the spacecraft potential. Indeed, the spacecraft potential estimated from the energy shift of two spectral lines of the $\mathrm{CO}_{2}$ photoelectrons, seen on the spectrograms of the electron fluxes (the second panels), is about of -8 to $-9 \mathrm{~V}$ that permit to detect the core of the cold ionospheric plasma. The black and red curves on the top panels depict the electron number density measured by MARSIS and the total number of counts measured in the 'oxygen channel' for $E_{\mathrm{i}} \leq 50 \mathrm{eV}$ by the IMA mass-spectrometer, respectively. Comparison of the measurements made by both instruments shows that 

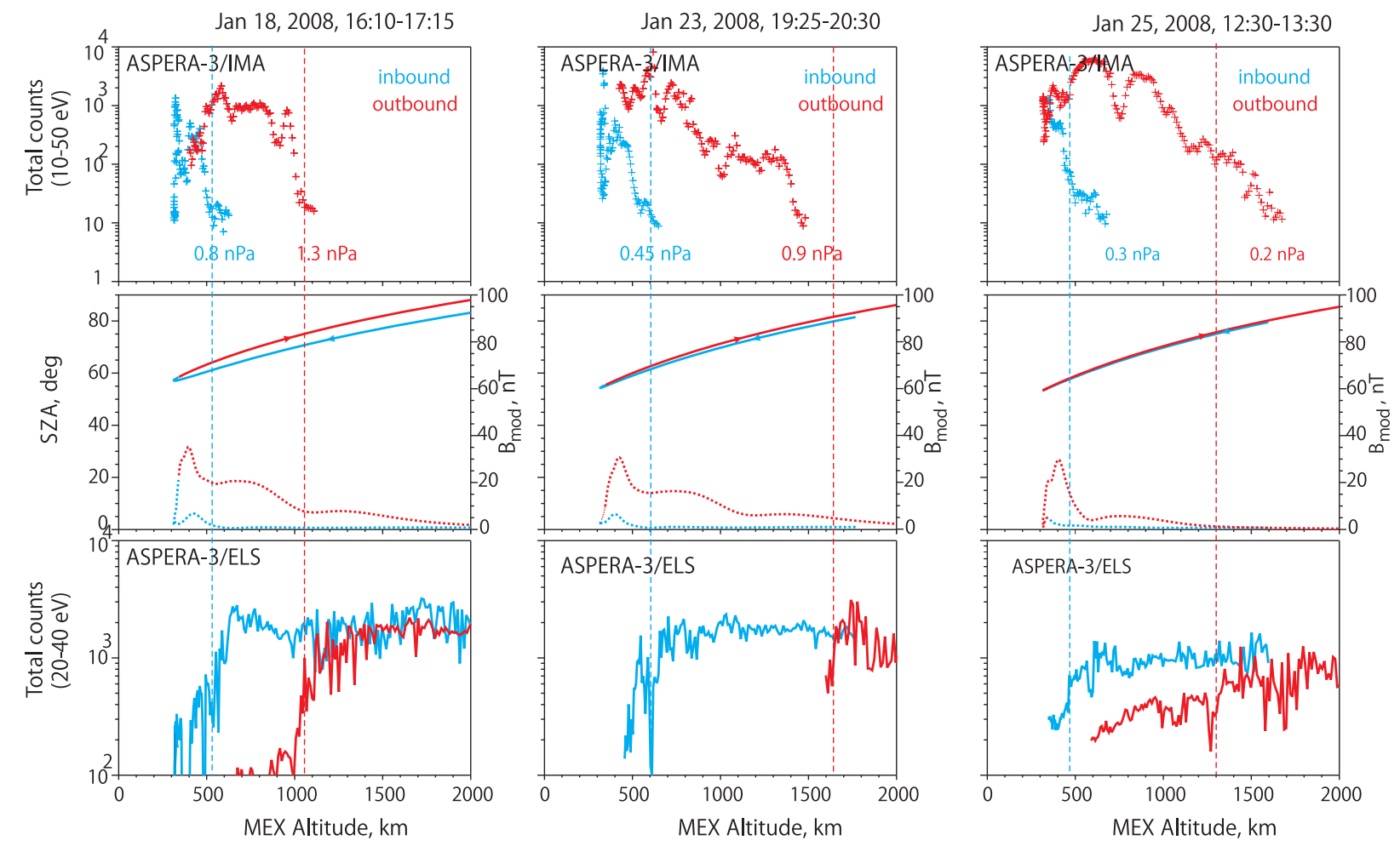

Fig. 4. From top to bottom: (a) total number of counts in the 'oxygen channel' $\left(E_{\mathrm{i}} \leq 50 \mathrm{eV}\right)$ on the inbound and outbound legs, (b) solar zenith angle and crustal magnetic field, (c) fluxes of the magnetosheath electrons.

the IMA data can be used as an additional and complementary tool to study the ionospheric plasma (see more details in Dubinin et al., 2008b; Fraenz et al., 2010). Further, in cases when the MARSIS data are unavailable, the measurements of low-energy oxygen ions will be used as a proxy for ionospheric density. Asymmetry between the inbound and outbound legs which are easily recognized from the pericenter position (red dotted curves on the second panels depict the altitude of MEX) is well seen. The extent of the outbound (inbound) ionosphere is higher on the orbits on Nov. 26 (Nov. 19). The MARSIS and IMA data plotted as a function of the altitude (third and fourth panels) clearly show this asymmetry.

Similar asymmetry between the inbound and outbound crossings is seen in Fig. 4 which shows the altitude profiles of cold oxygen ions measured by IMA-ASPERA-3 on several orbits sampling the same solar zenith angles. The corresponding values of the solar wind dynamic pressures are also given. As in the previous examples, such an asymmetry can not be explained by the variations of solar wind dynamic pressure. Subsequently we will use the IMA data, which provide us a better statistics, to study possible mechanisms causing such an asymmetry.

\section{Discussion}

It is observed that the Martian ionosphere at altitudes $\geq 400 \mathrm{~km}$ is strongly azimuthally asymmetrical. Such an asymmetry is closely related to an asymmetry in the flow of the shocked solar wind around Mars. A flow asymmetry can appear due to either variations in the solar wind dynamic pressure or inherent features of solar wind/Mars interaction. For the reason that in many cases the observed asymmetry can not be explained by solar wind variations we consider other factors which might influence the upper ionosphere. The induced magnetosphere of Mars is produced by draping of the IMF. Asymmetry in the pile-up observed by Mars Global Surveyor (MGS) (Vennerstrom et al., 2003) can lead to a more effective screening of the ionosphere in the hemisphere in which the motional electric field is pointed outward the planet (the $E^{+}$hemisphere). On the other hand, the forces due to the normal and tangential magnetic field tensions driving the planetary plasma into motion are also stronger in the $E^{+}$hemisphere. It will lead to scavenging of the ionospheric plasma, also extraction of the ionospheric ions by the $-\boldsymbol{V}_{\mathrm{sw}} \times \boldsymbol{B}$ electric field, and, as the effect, to the ionospheric depletion. Therefore it is not clear what processes will prevail for producing an asymmetry.

Since there is no magnetometer on MEX, the vector of the spacecraft position relative to the vectors $\boldsymbol{B}_{\mathrm{IMF}}$ and $\boldsymbol{E}$ could be determined only as a proxy from the MGS measurements on the mapping orbits at $h \sim 400 \mathrm{~km}$ in some reference point in the northern hemisphere and then adjusted to the MEX observations (Brain et al., 2006; Dubinin et al., 2006). Although this method is rather crude since the orbital period of MGS is about 2 hours and the IMF orientation often significantly varies during such time we try to use it plotting the inbound and outbound orbital legs for 12 orbits sampling the same solar zenith angles as a function of the $Z_{\mathrm{IMF}}$ (Fig. 5(a)). Here the $Z_{\mathrm{IMF}}$ axis is along the motional electric field in the solar wind. Red and blue segments of the orbital trajectories correspond to the inbound and outbound paths on which the ionosphere was expanded or contracted, respectively. There is no evident asymmetry between the $E^{+}$and $E^{-}$hemispheres. 
a)

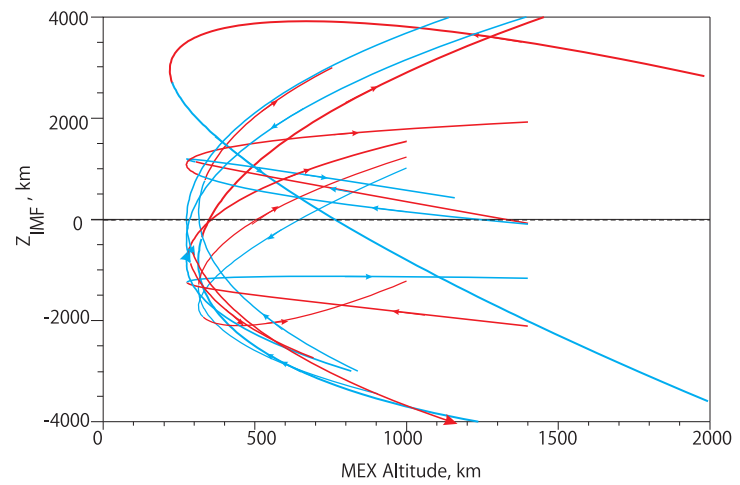

b)

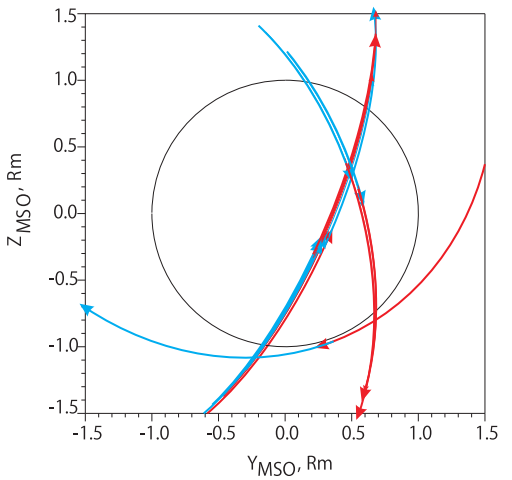

c)

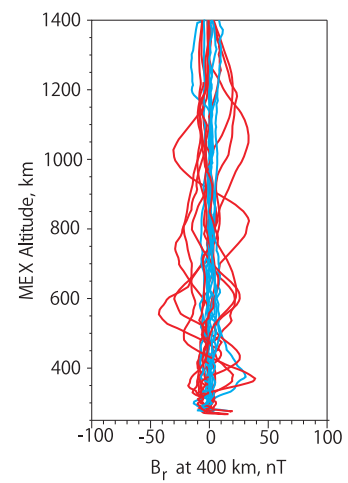

Fig. 5. Trajectories of inbound and oubound parts of selected 12 MEX orbits on which MARSIS have observed a distinct asymmetry in the height ionospheric density profiles. Red and blue curves correspond to the trajectories along which the expanded or contracted ionosphere was respectively observed. Trajectories are plotted in the variables: (a) Altitude- $Z_{\mathrm{IMF}}$, (b) $Y Z_{\mathrm{MSO}}$ and (c) Altitude-Strength of crustal field at $400 \mathrm{~km}$ inferred from the Cain model.
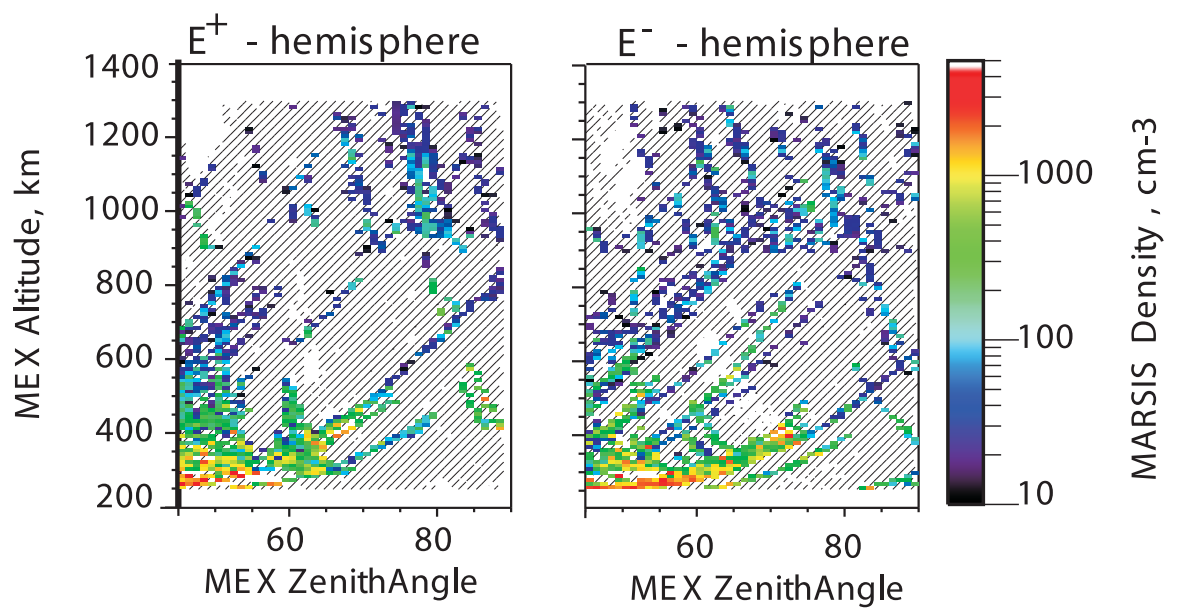

Fig. 6. Maps of the mean values of the electron number density in the bin $40 \mathrm{~km} \times 1^{\circ}$ as a function of altitude and solar zenith angle. Black shaded bins have values lower or equal than the low threshold of the MARSIS instrument $\left(n_{\mathrm{e}} \sim 10-20 \mathrm{~cm}^{-3}\right)$.

Figure 6 compares the maps of the local electron number density measured by MARSIS in the $E^{+}$and $E^{-}$hemispheres for solar zenith angles $45^{\circ} \leq \mathrm{SZA} \leq 90^{\circ}$ during years 2005-2006 (MGS was lost in November 2006). An asymmetry, if it does exist, is very weak although the statistics are also not sufficient.

In spite of the absence after November 2006 of the magnetic field measurements near Mars, a certain knowledge of the global magnetic field configuration can be retrieved from the ELS and IMA data. For example, in Fig. 3, the crossing of the plasma sheet can be recognized from the electron spectrograms - a narrow region at $\sim 12: 46 \mathrm{UT}$ (November 19) and a broader region centered approximately at $\sim 12: 48$ UT (November 26). This gives us the approximate position of the plasma sheet in the $Y Z \mathrm{MSO}$ reference frame which coincides with the direction of the motional electric field $\left(-\boldsymbol{V}_{\mathrm{sw}} \times \boldsymbol{B}_{\mathrm{IMF}}\right)$ in the induced magnetosphere. The uncertainty in the sign of the $\boldsymbol{E}$ vector can be solved from the ion measurements. An increase in the energy of the outflowing oxygen ions with a distance from Mars observed at 13:10-13:20 UT (Nov. 19) and 12:3512:50 UT (Nov. 26) identifies the $E^{+}$hemisphere with outward directed electric field. Figure 7 shows these two orbits and the directions of the cross-flow component of the IMF and the motional electric field in the $Y Z$ MSO plane. Blue and red segments correspond to the inbound and outboung legs, respectively. For the orbit on Nov. 26, the ionosphere is more extended in the $E^{-}$hemisphere, on the orbit on Nov. 19, the asymmetry is rather observed in the magnetic equatorial plane.

The latter asymmetry can appear due to the Parker orientation of the IMF in the solar wind. For the nominal IMF orientation $(+Y-X$ or $-Y+X)$ the induced magnetosphere is stronger protected from the magnetosheath plasma at the dusk side where the draped magnetic field is almost tangential to the magnetospheric boundary while at the dawnside, where the magnetic field is more radial, solar wind more easy gain access to the magnetosphere (Dubinin et al., 2008c). Figure 5(b) shows the orbital segments on which the ionosphere was expanded (red curves) or contracted (blue curves) in the $Y Z_{\text {MSo }}$ plane for the 12 selected cases. It is worth noting that the MEX orbit is not suitable to study a dawn-dusk asymmetry because the spacecraft surveyed mainly the low-altitude dusk ionosphere. It is well seen in Fig. 10(a) which shows the map of the fluxes of the low-energy oxygen ions in the $X Y_{\text {MSO }}$ plane. The absense 

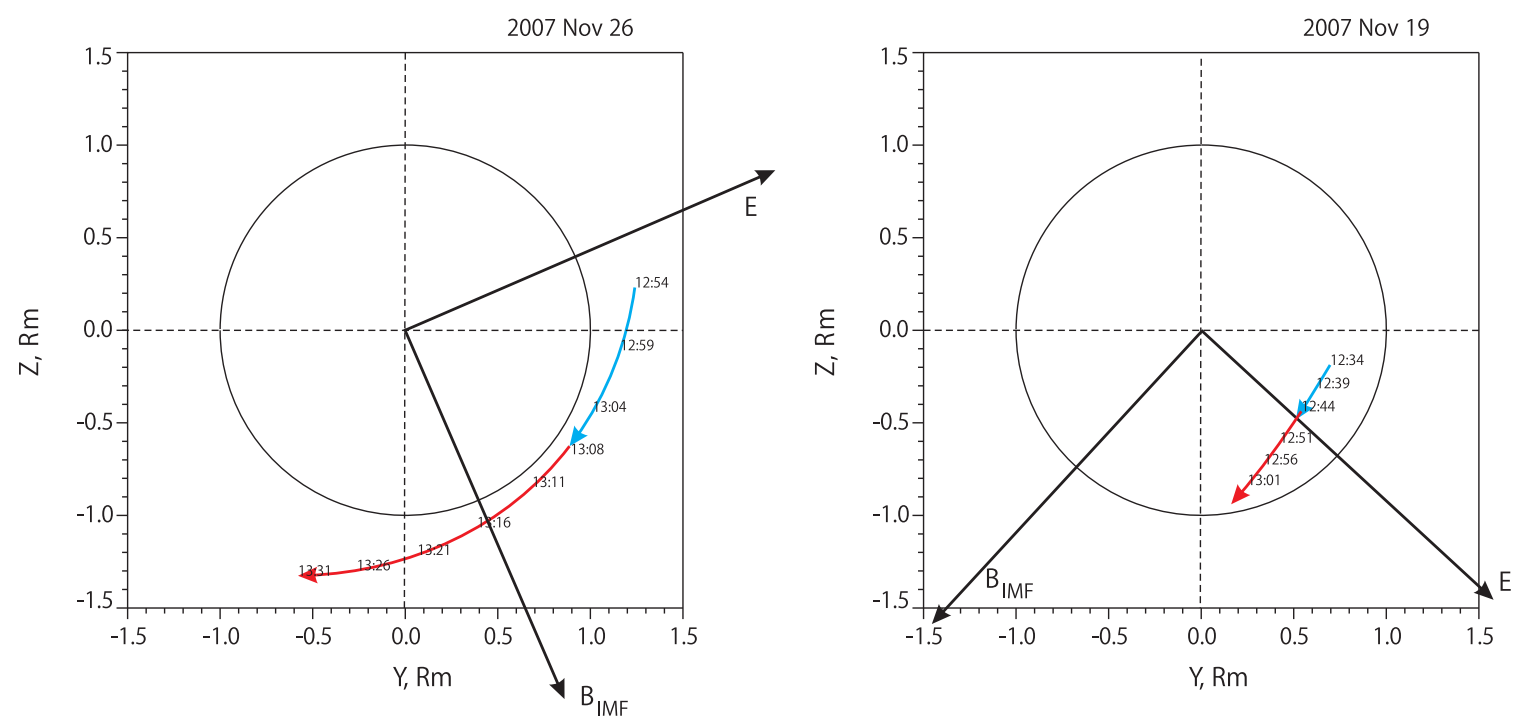

Fig. 7. Trajectories of the inbound (blue) and outbound (red) parts of the MEX trajectories on Nov. 26 and 19, 2007 (see Fig. 3 ) and the directions of the cross-flow component of the IMF and the motional electric field in the $Y Z_{\text {MSO }}$ plane.

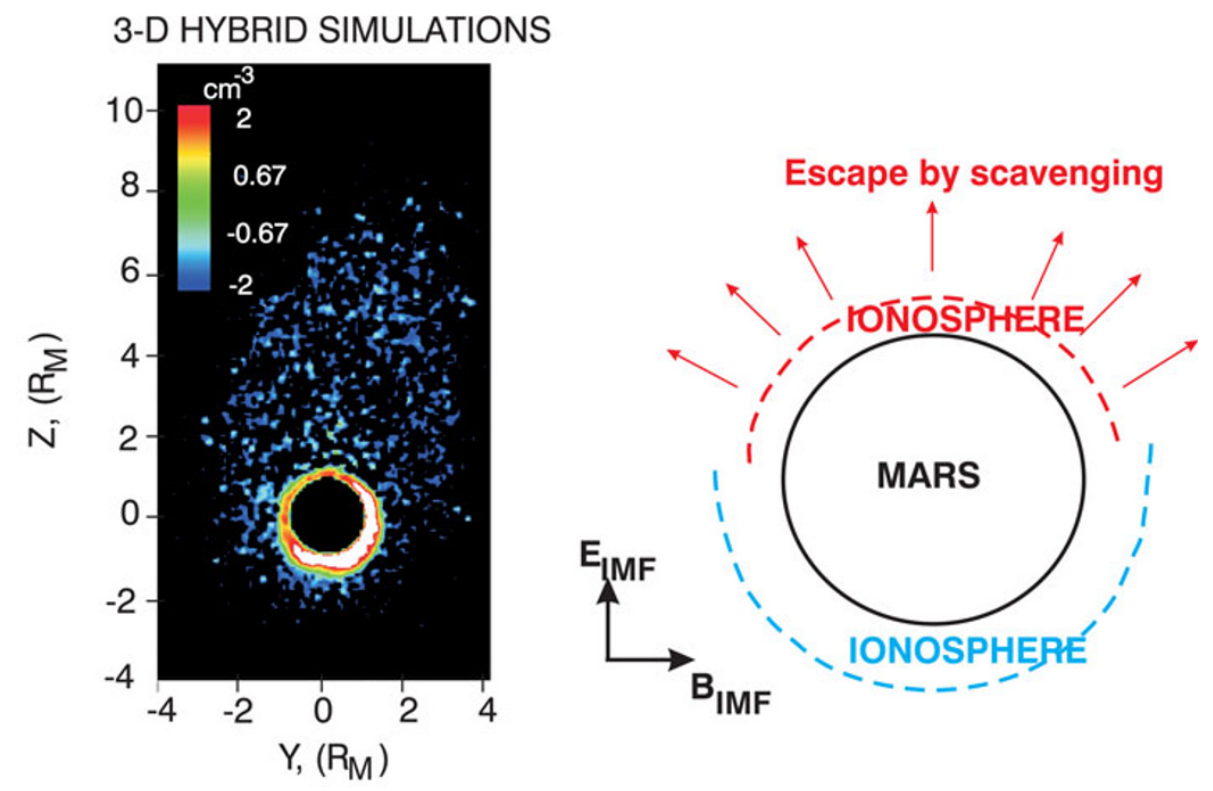

Fig. 8. Map of the $\mathrm{O}^{+}$number density in the $Y_{\mathrm{IMF}} Z_{\mathrm{IMF}}$ plane near the terminator obtained from hybrid simulations. In white bins the number densities are above $10^{2} \mathrm{~cm}^{-3}$.

of ion fluxes at the dawn side is due to the fact that MEX sampled this region at much higher altitudes.

Since the absence of a magnetometer and proper MEX orbital sampling makes the retrieval of an asymmetry which may be caused by the motional electric field and the Parker IMF orientation more difficult, we have also performed 3D hybrid and multi-ion species simulations (see Modolo et al., 2005 for details). Figure 8 shows the distribution of oxygen ions in the $Y_{\mathrm{IMF}} Z_{\mathrm{IMF}}$ plane near the terminator. It is seen that the upper ionosphere is filled by oxygen ions extracted by the $-\boldsymbol{V}_{\mathrm{sw}} \times \boldsymbol{B}$ electric field. On the other hand, the $E^{-}$ionosphere occurs inflated as compared to the $E^{+}$hemisphere in which ionospheric ions are emitted into space. The asymmetry probably appears due to the recoil effect of a dense ionospheric plasma on extraction of ions in the $E^{+}$hemisphere to conserve the transverse momentum of the system. However the details of such a process as well as forces exerting on the ionospheric plasma remain unclear. Szego et al. (2000, page 621) have suggested the existence in a dense plasma of a small polarization electric field pointed in the $E^{-}$direction and pushing the whole ionosphere in the $E^{-}$direction. A possible asymmetry related to the Parker IMF orientation was not observed in the $3 \mathrm{D}$ hybrid simulations.

Asymmetry of plasma flow can also appear due to crustal magnetic field. Crider et al. (2002) have found that the magnetospheric boundary moves upward with increasing southern latitude. Fraenz et al. (2006) and Dubinin et al. (2008a) have shown that the boundary above strong crustal sources can shift upward by $\sim 400 \mathrm{~km}$ as compared to the boundary in the northern hemisphere. Due to the local origin of crustal magnetic fields on Mars, the surface of the mag- 
a)
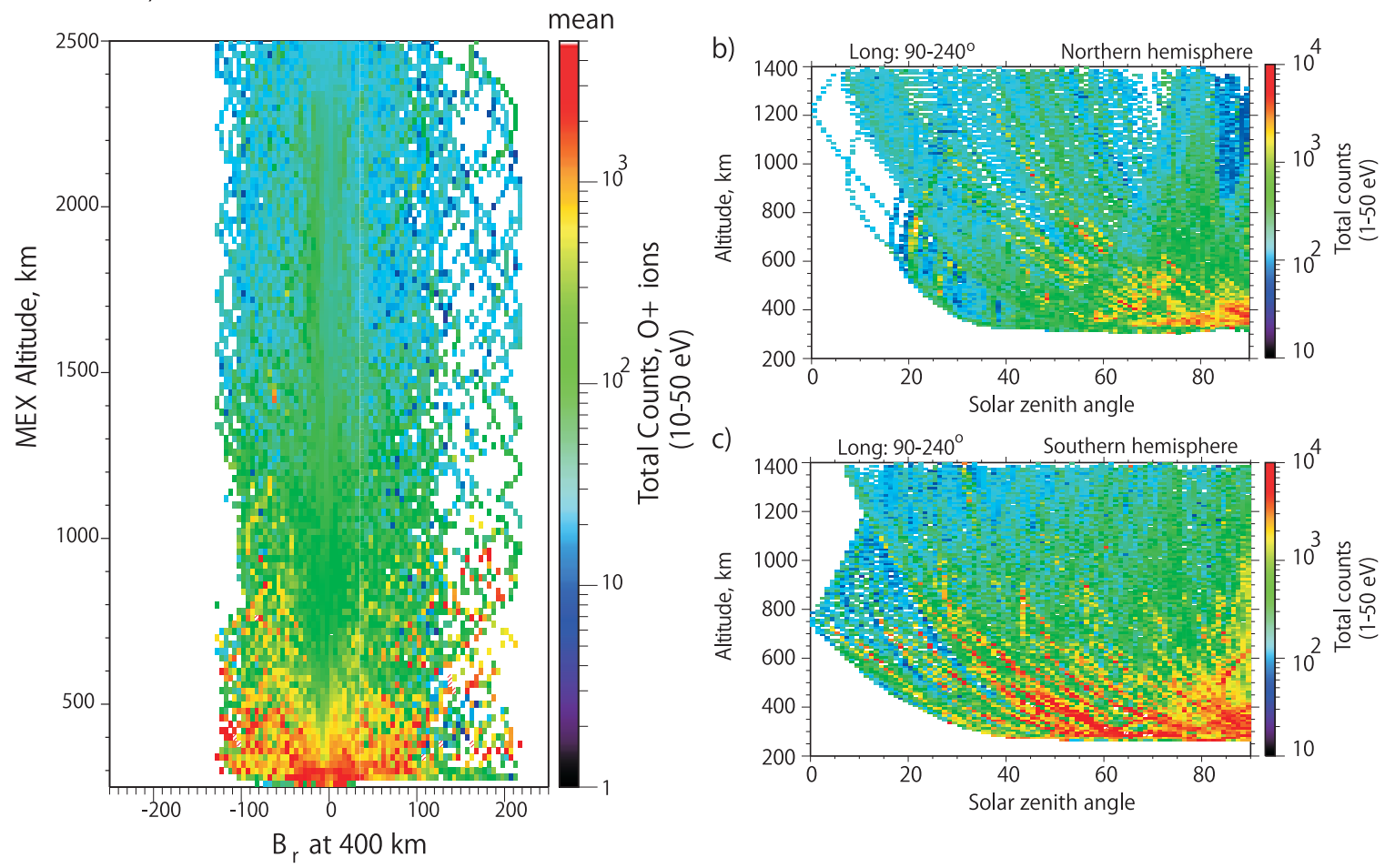

Fig. 9. (a) Mean fluxes of low-energy ionospheric oxygen ions measured by IMA during 3 years of MEX observations are plotted as a function of the altitude and the strength of crustal magnetic field. (b, c) 'Ionospheric' maps measured by IMA in the northern and southern hemispheres at the geographic longitudes $90^{\circ}-240^{\circ}$, respectively.
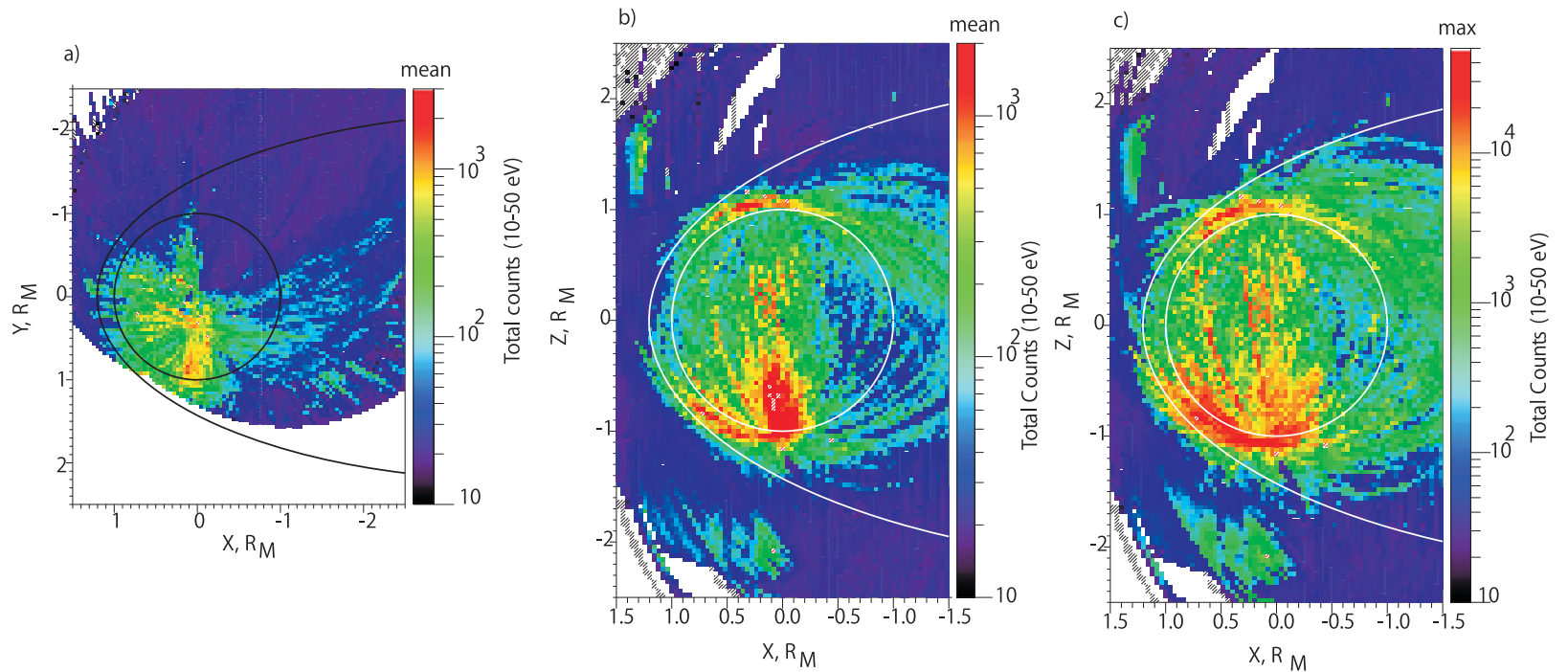

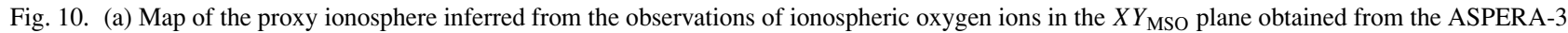
data during almost 3 years of the observations. Strong asymmetry is caused by the fact that the dawn ionosphere was sampled at much higher altitudes than the ionosphere at the dusk side. (b, c) Maps of mean and maximum numbers of counts of low-energy oxygen ions in the $X Z_{\mathrm{MSO}}$ plane. Strong asymmetry between the northern and southern ionospheres appears due to the crustal magnetic field. Note that the high fluxes measured in the solar wind are mainly due to the UV contamination.

netospheric cavity occurs corrugated (Brain et al., 2006; Dubinin et al., 2008c) and the ionospheric plasma lifting up along the crustal field lines produces swells in the density distribution. Figure 5(c) shows the trajectories of MEX orbits at which MARSIS examined the upper ionosphere at almost the same solar zenith angles on the inbound and outbound crossings. Blue and red curves correspond to the orbital legs at which the ionosphere was contracted or ex- panded, respectively. Trajectories are given in the variables: altitude-model radial component of the crustal magnetic field at $400 \mathrm{~km}$. It is seen that although for these selected cases we try to minimize a possible role of crustal sources, the ionospheric expansion usually occurs on the orbital segments with higher values of the crustal field, even if the value of the local field is small to balance the solar wind dynamic pressure. 
Figure 9(a) presents the data of the IMA monitoring of the upper ionosphere during almost 3 years of the MEX observations. The mean fluxes of cold and low energy $\left(E_{\mathrm{i}} \leq 50 \mathrm{eV}\right)$ ionospheric oxygen ions are plotted as a function of the same variables. The observed upward lifting of oxygen ions in the regions with strong crustal magnetization can be responsible for a significant asymmetry of the upper ionosphere (see also Lundin et al., 2011).

The importance of this factor is also illustrated in Fig. 9(b, c) which compares the maps of the fluxes of low-energy ionospheric oxygen ions as a function of solar zenith angle and the MEX altitude obtained from the IMA-ASPERA-3 measurements performed in the northern and southern hemispheres in the interval of geographic longitudes in the range of $90^{\circ}-240^{\circ}$. A clear asymmetry between the northern hemisphere where the pure induced magnetosphere is formed and the southern hemisphere with strongest local magnetization where crustal magnetic field essentially contribute to the solar wind/Mars interaction is observed.

It seems that the crustal magnetic field localized mainly in the southern hemisphere globally influences the interaction pattern increasing the altitude of the obstacle and lifting upward the ionospheric plasma. Figure $10(\mathrm{~b}, \mathrm{c})$ clearly illustrates this point. It shows the maps $\left(X Z_{\mathrm{MSO}}\right.$ plane) of mean and maximum numbers of counts of the lowenergy oxygen ions measured in each bin during 3 years of ASPERA-3 observations. It is observed that the southern ionosphere spreads to higher altitudes as compared to the northern one. Although the case studies show that asymmetry in the local model crustal field along the orbits can be rather small, the global increase in the size of the magnetospheric obstacle above the regions with crustal field enlarges the upper ionosphere.

\section{Conclusions}

A distinct asymmetry between the altitude profiles of the Martian ionosphere on the inbound and outbound parts of the MEX orbits inferred from the in-situ measurements of the plasma density by ASPERA-3 and MARSIS is observed. It is shown that such an asymmetry is accompanied by the asymmetry in the solar wind flow around Mars. The asymmetry can appear not only due to variations in solar wind dynamic pressure but also due to the inherent features of the solar wind/Mars interaction. In particular, the ionosphere over the regions with strong crustal magnetization occurs more inflated due to a lift of plasma along the crustal magnetic field lines. A possible asymmetry caused by the motional electric field can be disguised by errors in the determination of its direction. However the 3D hybrid simulations reveal such an asymmetry-the ionosphere in the $E^{-}$hemisphere is more swelled as compared to the $E^{+}$ hemisphere. A possible dawn-dusk asymmetry due to the Parker IMF could not be studied because of inappropriate dawn-dusk ionosphere sampling by the MEX spacecraft.

Acknowledgments. E. D., M. F. and J. W. wish to acknowledge the DLR and DFG for supporting this work by grants FKZ 50 QM 0801, O539/17-1 and DFG-grant SPP 1488 W0910/3-1, respectively.

\section{References}

Barabash, S., R. Lundin, H. Andersson et al., The analyzer of space plasma and energetic atoms (ASPERA-3) for the Mars Express mission, Space Sci. Rev., 126, 113-164, 2006.

Brain, D. A., J. S. Halekas, R. J. Lillis et al., Variability of the altitude of the martian sheath, Geophys. Res. Lett, 32, doi:10. 1029/2005GL023126.L18203, 2006.

Cain, J. C., B. Ferguson, and D. Mozzoni, An n=90 internal potential function of the martian crustal magnetic field, J. Geophys. Res., 108, doi:10.1029/2000JE001487, 2003.

Crider, D. H. et al., Observations of the latitude dependence of the location of the martian magnetic pileup boundary, Geophys. Res. Lett, 29, 11, 2002.

Dubinin, E., M. Fraenz, J. Woch et al., Plasma morphology at Mars. ASPERA-3 observations, Space Sci. Rev., 126, 209-238, 2006.

Dubinin, E., M. Fraenz, J. Woch et al., Access of solar wind electrons into the Martian magnetosphere, Ann. Geophys., 26, 3511-3524, 2008a.

Dubinin, E., R. Modolo, M. Fraenz et al., Plasma environment of Mars as observed by simultaneous MEX-ASPERA-3 and MEX-MARSIS observations, J. Geophys. Res., 113, A10217, doi:10.1029/2008JA013355, $2008 b$.

Dubinin, E., G. Chanteur, M. Fraenz et al., Asymmetry of plasma fluxes at Mars. ASPERA-3 observations and hybrid simulations, Planet. Space Sci., 56, 832-835, 2008c.

Duru, F., D. A. Gurnett, D. D. Morgan et al., Electron densities in the upper ionosphere of Mars from the excitation of electron plasma oscillations, J. Geophys. Res., 113, A07302, doi:10.1029/2008JA013073, 2008.

Fraenz, M., J. D. Winningham, E. Dubinin et al., Plasma intrusion above Mars crustal fields-Mars Express ASPERA-3 observations, Icarus, 182, 406-412, 2006.

Fraenz, M., E. Dubinin, E. Nielsen et al., Transterminator ion flow in the Martian ionosphere, Planet. Space Sci., 58, 1442-1454, 2010.

Gurnett, D. A., D. L. Kirchner, R. L. Huff et al., Radar sounding of ionosphere of Mars, Science, 310, 1929-1933, 2005.

Gurnett, D. A., R. L. Huff, D. D. Morgan et al., An overview of radar soundings of the martian ionosphere from the Mars Express spacecraft, Adv. Space Res., 41, 1335-1346, 2008.

Hanson, W. C., S. S. Sanatani, and D. R. Zuccaro, The martian ionosphere as observed by the Viking retarding potential analyzers, J. Geophys. Res., 82, 4351, 1977.

Hanson, W. B. and G. P. Mantas, Viking electron temperature measurements: evidence for a magnetic field in the Martian ionosphere, J. Geophys. Res., 93, 7538, 1988.

Kliore, A. J., Radio occultation observations of the ionospheres of Mars and Venus, in Venus and Mars: Atmospheres, Ionospheres and Solar wind Interactions, Geophys. Monogr. Ser. vol. 66 , edited by Luhmann, J. G., M. Tatrallyay, and R. O. Repin, 265-276 pp, AGU, Washington, 1992.

Lillis, R. J., D. Brain, S. L. England et al., Total electron content in the Mars ionosphere: Temporal studies and dependence on solar EUV flux, J. Geophys. Res., 115, A11314, doi:1029/2010JA015698, 2010.

Lundin, R., S. Barabash, T. Yamauchi, N. Nilsson, and D. Brain, On the relation between plasma escape and the Martian crustal magnetic field, Geophys. Res. Lett., 38, L08102, doi:10.1020/2010GL046019, 2011.

Mendillo, M., P. Withers, D. Hinson et al., Effects of solar flares on the ionosphere of Mars, Science, 311, 1135-1138, 2004.

Modolo, R., G. Chanteur, E. Dubinin, and A. P. Matthews, Influence of the solar EUV flux on the Martian plasma environment, Ann. Geophys., 23, 433-444, 2005.

Morgan, D. D., D. A. Gurnett, D. L. Kirchner et al., Variations of Mars ionospheric electron density from Mars Express radar sounding, J. Geophys. Res., 113, A09303, doi:10:1029/2008JA013313, 2008.

Pätzold, M., S. Tellmann, B. Häusler et al., A sporadic third layer in the ionosphere of Mars, Science, 310, 837-839, 2005.

Szego, K., K.-H. Glassmeier, R. Bingham et al., Physics of mass-loaded plasmas, Space Sci.Rev., 94, 623, 2000.

Vennerstrom, S., N. Olsen, M. Purucker et al., The magnetic field in the pileup region of Mars and its variation with solar wind, Geophys. Res. Lett., 30, 1369, doi:10.1029/2003GL016883, 2003.

Withers, P., A review of variability in the dayside ionosphere of Mars, $A d v$. Space Res., 44(3), 277-307, 2009.

E. Dubinin (e-mail: dubinin@mps.mpg.de), M. Fraenz, J. Woch, R. Modolo, G. Chanteur, F. Duru, D. A. Gurnett, S. Barabash, and R. Lundin 